E3S Web of Conferences 1, 20002 (2013)

DOI: $10.1051 / \mathrm{e} 3$ sconf/20130120002

(c) Owned by the authors, published by EDP Sciences, 2013

\title{
Seasonal variations in the concentration and solubility of elements in atmospheric particulate matter: a case study in Northern Italy
}

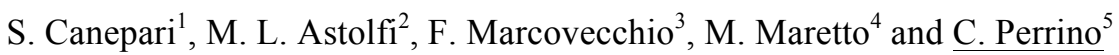 \\ ${ }^{1}$ Department of Chemistry, Sapienza University of Rome, P.le Aldo Moro 5, 00185, Rome, ITALY, \\ silvia.canepari@uniroma1.it \\ 2 Department of Chemistry, Sapienza University of Rome, P.le Aldo Moro 5, 00185, Rome, ITALY, \\ marialuisa.astolfi@uniroma1.it \\ ${ }^{3}$ Institute of Atmospheric Pollution Research, C.N.R., Via Salaria Km 29.300, 00015, Monterotondo St. (Rome), ITALY, \\ marcovecchio@iia.cnr.it \\ ${ }^{4}$ Department of Chemistry, Sapienza University of Rome, P.le Aldo Moro 5, 00185, Rome, ITALY, \\ moreno.maretto@uniroma1.it \\ ${ }^{5}$ Institute of Atmospheric Pollution Research, C.N.R., Via Salaria Km 29.300, 00015, Monterotondo St. (Rome), \\ ITALY, perrino@iia.cnr.it
}

\begin{abstract}
Atmospheric particulate matter is characterized by a variety of chemical components, generally produced by different sources. Chemical fractionation of elements, namely the determination of their extractable and residual fractions, may reliably increase the selectivity of some elements as tracers of specific $\mathrm{PM}$ sources. Seasonal variations of atmospheric particulate matter concentration in $\mathrm{PM}_{10}$ and $\mathrm{PM}_{2.5}$, of elemental concentration in $\mathrm{PM}_{10}$ and $\mathrm{PM}_{2.5}$, of the extractable and residual fraction of elements in different size fractions in the range $0.18-18 \mu \mathrm{m}$ are reported in this paper. The effect of the ageing of the air masses is discussed.
\end{abstract}

Key words: particulate matter, atmosphere, elements, chemical fractionation, size distribution.

\section{Introduction}

The study of the chemical composition of atmospheric particulate matter $(\mathrm{PM})$ is a fundamental step to obtain information about its sources and effects on both the human body and the ecosystem. PM is, in fact, composed of a wide variety of chemical species, ranging from macro-components, typically accounting for more than $1 \%$ of the total PM mass, to microcomponents and trace species. While organic species include hundreds/thousands of chemical species, generally present as micro-components and trace species, inorganic PM components include some macro-components, generally some ionic species and macro-elements (e.g.: silicon, iron, aluminum), as well as micro-elements (e.g.: copper, manganese, lead) and trace elements (e.g.: arsenic, cadmium, cobalt).

It is worth noting that only a few elements in atmospheric PM have a predominant origin, and for this reason they are precious tracers of the source they share. Crustal elements, for example, are able to trace the release of soil particles into the atmosphere. The majority of atmospheric elements, however, derive from multiple sources, and the identification of the relative contribution of these sources to atmospheric PM concentration in a given area and time is a demanding task, often attempted by means of statistical tools.

In this perspective, the study of additional chemical characteristics of elements can be of help. Chemical fractionation has proved to be a reliable tool for increasing the selectivity of some tracers of specific pollution sources and for obtaining rough information about their bio-availability and toxicity (Canepari et al., 2006, Perrino et al., 2008). We report in this paper some results obtained by determining the extractable and residual fractions of some trace elements in different size fractions of atmospheric PM during different times of the year.

\section{Materials and Methods}

Measurements were carried out in the area of Ferrara (Northern Italy), a site characterized by the presence of several PM sources, among which the urban area, the 
highway A13 and a wide industrial area including a power plant, a urban waste incinerator and many small and medium enterprises. From the meteo-climatic point of view the site is affected by long winter periods of strong atmospheric stability that causes ageing of the air mass, worsening of the air quality and relevant increase in the concentration of secondary pollutants.

Sampling of $\mathrm{PM}_{10}$ and $\mathrm{PM}_{2.5}$ was carried out by using SWAM 5a Dual Channel Monitors (FAI Instruments, Fonte Nuova, Rome - IT), which also yield the automatic measurement of the PM mass concentration by the beta attenuation method. PM size distribution was studied by using a 8-stage MOUDI impactor (mod. 100-R, MSP Co., USA).

In all cases PM was collected on Teflon membrane filters, $47 \mathrm{~mm}$ in diameter, $2 \mu \mathrm{m}$ pore size (PALL Corporation, U.S.A.).

Analytical determinations of elements were carried out by ICP-OES and ICP-MS (Varian Vista MPX and Varian 810, Mulgrave, Australia). According to the method reported by Canepari et al. (2006), sampled membranes were extracted in ultrasonic bath in acetate buffer solution at $\mathrm{pH} 4.5$, then the extracts were filtered on a cellulose nitrate filter and analyzed (extractable fraction); after this first step, both the cellulose nitrate filter and the original PTFE filter with the residue from the first stage were acid-digested in microwave oven and analyzed (mineralized residual fraction).

Sampling were carried out during eight 1-month Special Observation Periods (SOPs), during the winter (January) and the summer (June) of the years 2008-2011.

\section{Results and Discussion}

Figure 1 show the time variations of $\mathrm{PM}_{10}$ and $\mathrm{PM}_{2.5}$ average concentration during the eight 1-month SOPs. PM concentration during the winter is clearly higher than during the summer, for both size fractions. This difference is generally due to the poorer mixing of the atmosphere that is experienced during the winter period and to the presence of additional PM emission sources, such as domestic heating.

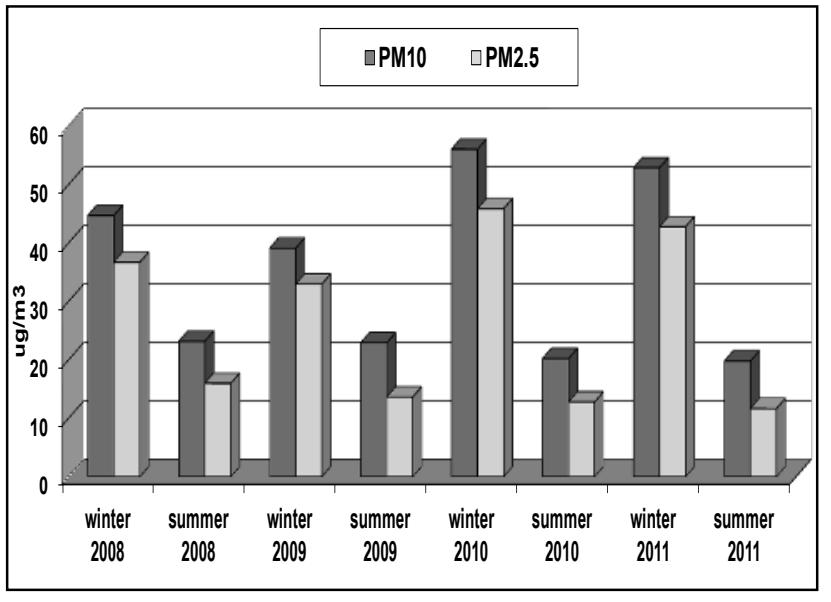

Fig. 1. Seasonal variations of the 1-month average concentration of $\mathrm{PM}_{10}$ and $\mathrm{PM}_{2.5}$.
Clear seasonal variations are also observed for elements. Some of them (e.g.: $\mathrm{Cd}, \mathrm{Pb}, \mathrm{Sn}$, and, at a less extent, $\mathrm{Ni}$ e $\mathrm{Sb})$ are distinctly higher during the winter. All these elements share anthropogenic origin and are mainly in the fine fraction $\left(\mathrm{PM}_{10} \approx \mathrm{PM}_{2.5}\right)$; their wintertime increase is due to both atmospheric stability and additional emission sources.

A second group of elements (Ti, Sr), of natural origin and mainly in the coarse fraction $\left(\mathrm{PM}_{10}>\mathrm{PM}_{2.5}\right)$, show the opposite behaviour: they increase during the summer because of soil drought, which enhance dust re-suspension. Figure 2 shows the seasonal pattern of cadmium, belonging to the first group, and titanium, belonging to the second one.

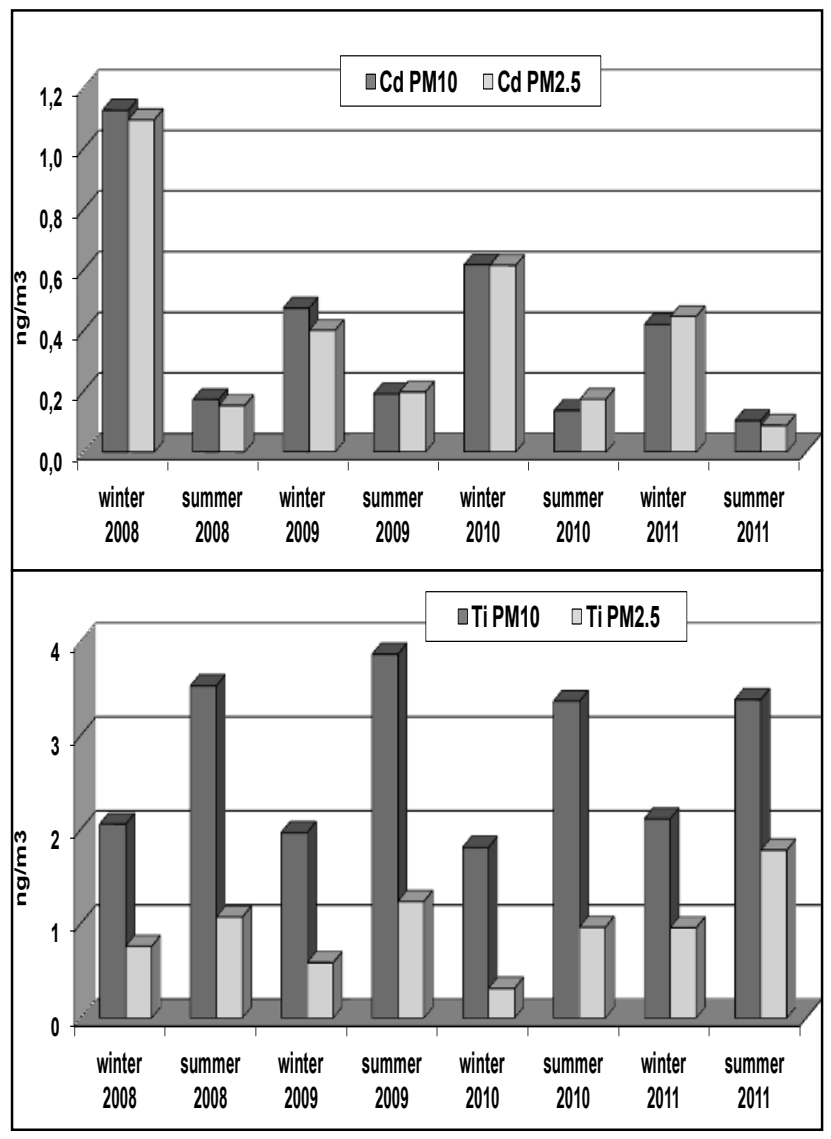

Fig. 2. Seasonal variations of the 1 -month average concentration of cadmium and titanium.

A third group of elements ( $\mathrm{Fe}, \mathrm{Mn}, \mathrm{Cu}, \mathrm{Li})$ show contributions from both natural sources, mainly in the coarse fraction, and anthropogenic sources, mainly in the fine fraction. In these cases the seasonal behavior is more complex: the fine fraction increases during the winter, while the coarse fraction increases during the summer.

More detailed information can be obtained from the chemical fractionation. For all elements showing a clear seasonal variation, this variation is generally associated to one of the two solubility fractions. In the case of $\mathrm{Cd}$, for example, the variation is predominantly associated to the extractable fraction, while in the case of $\mathrm{Ni}$ and $\mathrm{Pb}$ it is associated to the residual fraction. In the case of $\mathrm{Li}$, the extractable fraction is responsible for the winter increase 
while the residual fraction is responsible for the summer increase. These results indicate that the emission of these elements show a clear seasonal variability.

The extractable and residual fraction of almost all elements also show a different size distribution. Figure 3 reports, as an example, the dimensional distribution of litium in the size range between 0.18 and $18 \mu \mathrm{m}$. The data show that the extractable fraction is mostly in the fine size range, with a maximum between 0.32 and 0.56 $\mu \mathrm{m}$. The residual fraction, instead, is mostly in the coarse size range, with a maximum between 3.2 and $5.6 \mu \mathrm{m}$. The concentration of the total elemental content, without chemical fractionation, would have shown a more complex unresolved bi-modal distribution (bars in Figure 3).

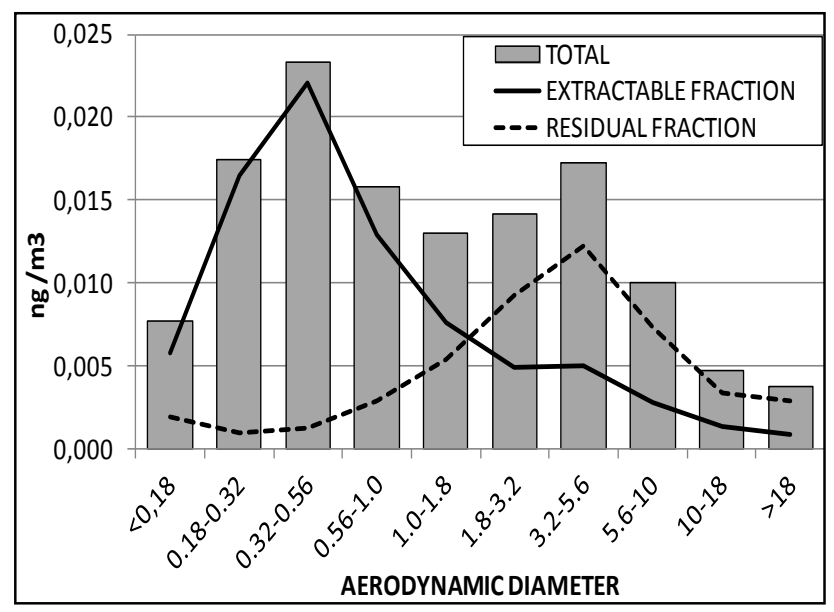

Fig. 3. Size distribution of the extractable and residual fractions of litium in atmospheric PM.

The period of the year also influences the size distribution of elements. The dimension of fine particles of anthropogenic origin tends to increase during the winter, while coarse particles, produced by abrasion, tends to decrease during the same period.

Both phenomena are linked to the ageing of the air masses, which is enhanced during the winter period. During their stay in the atmosphere, fine particles increase their dimensions because of condensation and coagulation processes, while coarse particles became smaller, as far as wind abrasion proceeds.

Figure 4 shows the different size distribution of the extractable and residual fractions of antimony during the winter and the summer periods.

\section{Conclusion}

Aging of the air masses may be responsible for variations in the size distribution of some elements, which may be highlighted by the use of chemical fractionation.

Seasonal differences in the concentration and composition of atmospheric PM can be interpreted on the

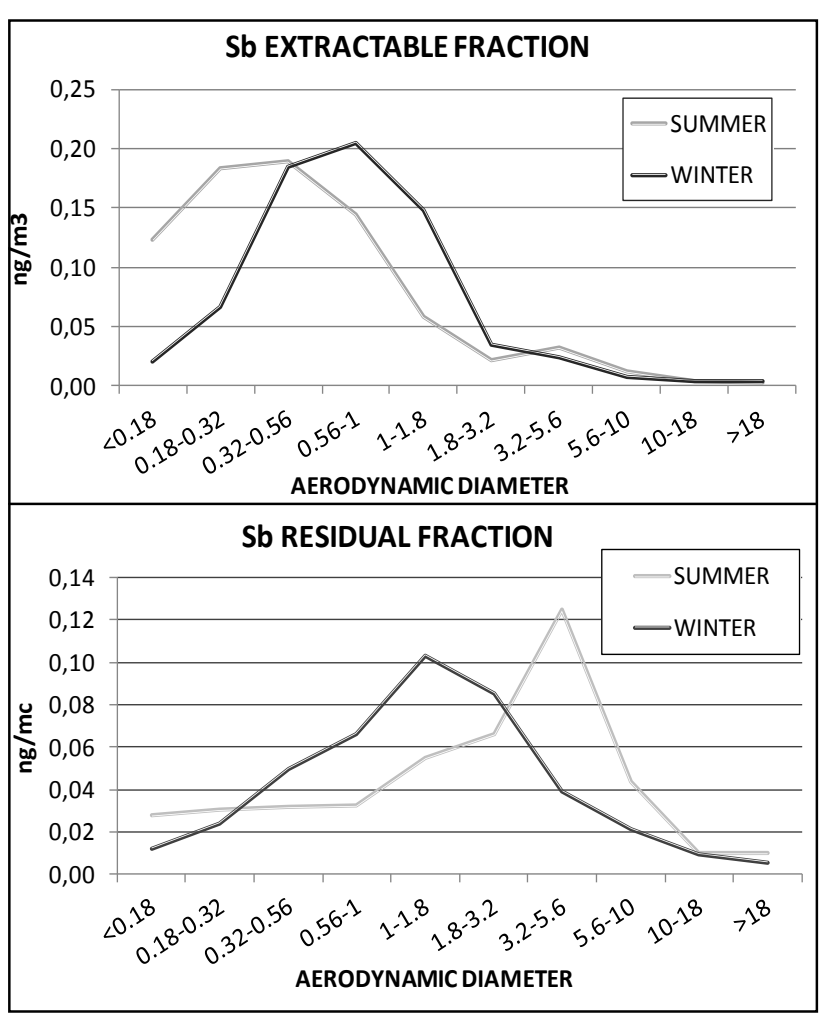

basis of variations in the strength of its manifold sources.

Fig. 4. Seasonal variation in the size distribution of the extractable and residual fractions of antimony in atmospheric PM.

\section{References}

Canepari S, Cardarelli E, Pietrodangelo A, Giuliano A. Determination of metals, metalloids and non-volatile ions in airborne particulate matter by a new two-step sequential leaching procedure. Part A: experimental desgn and optimization. Talanta 2006; 69:581-587.

Perrino C., Canepari S., Cardarelli E., Catrambone M., Sargolini T. Inorganic constituents of urban air pollution in the Lazio region (Central Italy). Environmental Monitoring and Assessment 2008; 136: 69-86. 